\title{
Modelling and Hazard Analysis of Sandia National Laboratories/Underwriters Laboratories' Experiments
}

DUY O. DUONG

Commonwealth Scientific and Industrial Research Organisation

Division of Building, Construction and Engineering

P.O. Box 310, North Ryde, N.S.W. 2113, Australia

\begin{abstract}
This study focuses on modelling experiments carried out by the Sandia National Laboratories/Underwriters Laboratories. The four computer fire models used for the study are FIRST, BRI2 (and its earlier version BRI1), FAST and COMPBRN III. The analysis shows that for those experiments simulated as gas burner fires, FIRST (with the Hasemi and McCaffrey plumes), BRI1 and COMPBRN III give reasonable agreement with the experiments. FAST seems to overestimate the hot layer temperature, although not to the same extent as BRI2. It is also attempted to model the fires as pool fires with FIRST; the results show marked deviations from the experimental results, which suggests further study on modelling of pool fires is required. However, the depth of the hot gas layer, the mass of hot gases flowing out through the doorway and the mass loss rate of fuel were not recorded in the experiments. These important parameters would have otherwise clarified many points and would significantly further our understanding of the fire behaviour and the modelling effort. Suggestions for close cooperation between fire researchers and testing personnel are discussed with the hope that future exercises could be more effectively carried out.
\end{abstract}

Keywords: Fire Models, Experimental Data, Plume Models, Hazard Analysis.

\section{INTRODUCTION}

Recently, much effort has been focused on fire modelling. With advances in fire science, a number of computer fire models have been developed with different levels of sophistication and complexity [1-5] to predict fire development in buildings. Since not all 
fire phenomena are well-understood, all models have many inherent limitations and assumptions. Therefore a good knowledge of fire physics is required for a successful application. To validate the computer models, a number of experiments have been carried out [e.g 6,7], normally for comparison with a specific computer model. This analysis is the second in a series [8] which attempts to compare the results obtained from computer fire models with a set of experiments, in this case, with experiments carried out by the Sandia National Laboratories/Underwriters Laboratories (SNL/UL) in the U.S.A.

\section{EXPERIMENTAL}

The experiments were carried out by the Underwriters Laboratories for the Sandia National Laboratories. The purpose of the experiments was to establish the validity of the claim that, in the event of a fire, a 6.1 metre separation without intervening combustibles would be sufficient to ensure that at least one tray of cables would remain functional in a nuclear power plant compartment. Data from four of the experiments has been compared with computational results from COMPBRN III by Ho et al [4].

The room configurations and experimental set-up are shown in Figure 1. Two horizontal cable trays, located $0.305 \mathrm{~m}$ from the ceiling but above the doorway, were separated from a heptane pool fire by a horizontal distance of at least $6.1 \mathrm{~m}$. Instruments were placed throughout the compartment and within the cable trays to measure the external heat flux and the temperature of the hot gases and the cable jackets.

\section{COMPUTER SIMULATIONS}

The models used in this analysis are

FIRST [1], a member of the Harvard family originally developed at Harvard University, Cambridge, U.S.A, and later enriched at the Center for Fire Research, National Institute of Standards and Technology, Gaithersburg, U.S.A;

BRI2, and its earlier version, BRI1 [5], developed at the Building Research Institute, Tsukuba, Japan;

FAST, version 18 [3], developed at the above Center for Fire Research; and COMPBRN III [4], developed at the University of California at Los Angeles, U.S.A.

Discussion of fire physics and the limitations as well as the computational technique employed in each model is beyond the scope of this paper and interested readers are referred to the above references.

The simulations used information from Figure 1 and the parameters listed in Table 1 for building geometry, building material, fuel and vent characteristics. However, since each model has its own limitations, a number of modifications to input files were necessary. 


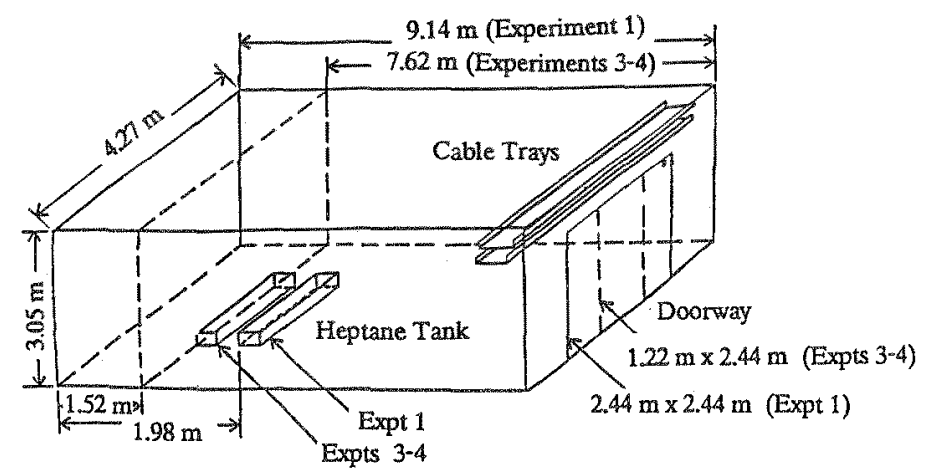

Figure 1: Enclosure Details of SNL/UL Experiments

TABLE 1. Parameters used in COMPBRN III, FIRST, BRI1, BRI2 and FAST

$\begin{array}{lllc}\text { Fire size } & (1400 \mathrm{KW}(\text { Expt 1) } & \text { Ambient temperature } & 290 \mathrm{~K} \\ & (1200 \mathrm{KW}(\text { Expts 3,4) } & \text { Heptane fire height } & 0.31 \mathrm{~m} \\ & & \text { Combustion efficiency } & 0.97\end{array}$

Ignition temperature for the cable jacket : $789 \mathrm{~K}$ (COMPBRN III and FIRST only)

Building material: concrete

$\begin{array}{lccc}\text { Thermal conductivity }(\mathrm{W} / \mathrm{m} . \mathrm{K}) & 0.8 & \text { Emissivity } & 0.9 \\ \text { Specific heat }(\mathrm{J} / \mathrm{kg} \mathrm{K}) & 880.0 & \text { Thickness }(\mathrm{m}) & 0.2 \\ \text { Density }(\mathrm{kg} / \mathrm{m} 3) & 2200.0 & & \end{array}$

\section{FIRST only:}

Pool radius : $0.388 \mathrm{~m}$

Specific heat : $2192 \mathrm{~J} / \mathrm{kg} . \mathrm{K}$

Emissivity : 0.42

Heat of combustion : $48400 \mathrm{KJ} / \mathrm{kg}$
Thermal conductivity : $0.128 \mathrm{~J} / \mathrm{m} . \mathrm{K}$

Density : $670 \mathrm{~kg} / \mathrm{m} 3$

Air/fuel ratio : 15.18

Heat of vaporisation : $362.0 \mathrm{KJ} / \mathrm{kg}$

\section{COMPBRN III only:}

Plume entrainment coefficient (expt 1) 2.0 (Fire away from wall or corner)

Combustion efficiency for Heptane (expt 3) 1.5 (Fire along the wall)

Fraction of heat loss by radiation 0.65 (Expt 4)

$$
0.4
$$

BRI2 has three restrictions for simulating these heptane fires; it deals only with gas burner fires, it does not take into account the case of a fire along the wall (although BRI1 does), nor does it address the ignition of another object in the room (i.e the cables in this analysis). FAST's physics is similar to BRI2's, but takes into account fires along the wall. FIRST, on the other hand, treats multiple objects in a room and therefore can be used to assess the hazard problem associated with fires in the same manner as COMPBRN III; it also treats pool fires as well as gas burner fires. 
All models treat three modes of heat transfer. For a given fire, the plume model, which has been shown perhaps to be the most important factor in modelling fires [8], is not an easy choice. COMPBRN III uses the Zukoski et al plume model [9], but with modified entrainment factors (Table 1), to take into account other effects (door mixing, wall jet etc) based on works by Zukoski et al [9], and McCaffrey and Quintiere [10]. FAST uses the McCaffrey plume [11]. BRI2 uses the Zukoski et al plume [9], compared with BRI1 [5], which used the McCaffrey plume. FIRST offers a choice of plume models, including the Hasemi et al [12] and the Standard (Morton et al) [13].

The solution is obtained by solving the equations of conservation of energy and mass together with the equation of state. All models run very quickly on a $25 \mathrm{MHz}$, $80386 / 80387$ IBM compatible computer. COMPBRN III took only 10 seconds, other models took from 90 seconds to 3 minutes when simulating a 900 second real-time fire.

\section{RESULTS}

The computational results for three fire parameters for each experiment: hot gas layer temperature, hot gas layer thickness and temperature of the cable tray are compared with the experimental values in Figures 2 to 9. Two important parameters for the assessment of the accuracy of computer fire models, the rate of heat release (RHR) and the thickness of the hot layer, were not measured by SNL/UL [14]. Therefore, the RHR for all models was taken to be the same as that predicted by COMPBRN III for the gas burner simulations or that predicted by FIRST for the pool fire simulations. In general, results from BRI2 in particular, consistently showed significantly higher hot gas temperatures than recorded in experiments 1 and 3 for the whole simulation period. However, BRI1, together with FIRST (with the Hasemi and McCaffrey plumes) and COMPBRN III, gave reasonable agreement with the experiments. Both FIRST and COMPBRN III predicted that the cables would not ignite and were consistent with the experiments.

Experiment 1: The results are shown in Figures 2 to 5. Within FIRST, the Hasemi plume gave the best agreement with the experimental data for the hot gas temperature (within 3.6\%- $10 \%$ error) but the Zukoski et al plume predicted $40 \%$ higher. All other plumes (Point and Standard (not shown), McCaffrey) predicted the temperature somewhere between the Zukoski and the Hasemi results with the McCaffrey being the second best (Figure 3).

BRI1, COMPBRN III and FAST gave similar results with a range of error between 10 $25 \%$; Figure 3 also includes the hot gas temperature curve from [4] (85\% combustion efficiency). BRI2 model predicted very high gas temperatures. Figure 5 shows the prediction of the hot gas thickness with marked deviations between the models. For the cable temperature, all the models gave results similar to the hot gas temperature curves (Figure 4). FIRST's pool fire simulations all exceeded their equivalent gas burner case 
by up to $20 \%$ and therefore, showed marked deviations from the experimental results (Figures 3,4).

Experiment 3: The predictions (Figures 6 to 7) showed a similar pattern to the above except that, for gas burner case, FIRST (Hasemi and MacCaffrey plumes) slightly underpredicted the hot gas temperature and FIRST with the McCaffrey plume was the best performer (less than $5 \%$ error). For the cable temperature, FIRST and COMPBRN III both gave better agreement than in experiment 1 .

Experiment 4: Figures 8 and 9 show results when the doorway was blocked except for a $0.01 \mathrm{~m}$ assumed gap under the door. In this case, the temperatures of the hot gas and the cable recorded in the SNL/UL experiment were well above the results obtained from FIRST. COMPBRN III (with combustion efficiency $65 \%$ ), on the other hand, gave comparable values to SNL/UL data for the first 600 seconds. The BRI2 model did not work at all even though it worked for a $0.05 \mathrm{~m}$ gap under the door (by trial and error). FAST gave the lowest results (not shown).

\section{DISCUSSION}

\section{Experiments 1 and 3:}

COMPBRN III, carried out with the inflow coefficient set to 0.6 and to 0.7 with other parameters unchanged. gave virtually the same results. Therefore the effect of inflow coefficient is negligible. It gave good agreement for both hot gas and cable temperatures because it included an allowance for the blockage effect and door and jet mixing, thereby increasing the air entrainment rate and reducing the hot gas temperature.

Within FIRST, the McCaffrey plume always gave lower hot gas temperature and higher gas thickness than the Zukoski plume. Similar result could be expected for BRI2 if the MacCaffrey plume is used. It is noted that BRI1 also used the McCaffrey plume and gave good agreement with the data. As FIRST clearly predicts higher temperatures for the cable trays than COMPBRN III and the data in all cases, it can be considered to give a more conservative assessment of the hazard situation.

\section{Experiment 4:}

The results (Figures 8 and 9) do not seem promising, with all predictions showing that the fire fell well short of oxygen within 40 seconds. In the experiment, the time was 800 seconds. Both values are most likely in error as the room would have run out of oxygen in 150 seconds. This means the fire would not have been sustained for 800 seconds unless there had been a large opening in the room. FIRST seems to underpredict the amount of oxygen available and the associated subroutine needs to be checked. 


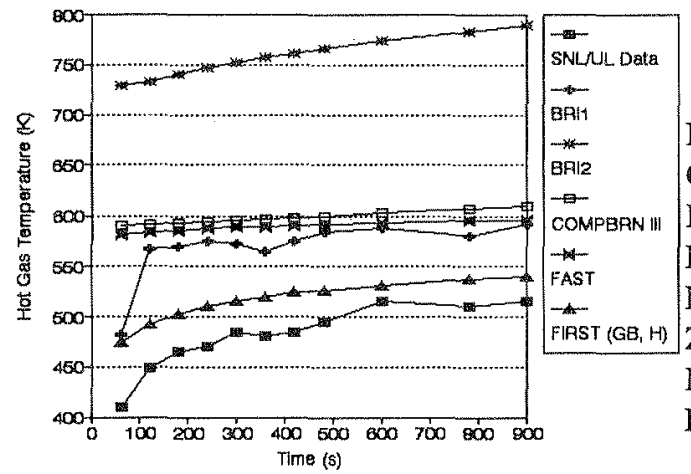

Notes:

GB: Gas Burner Fire

POOL: Pool Fire

$\mathrm{H}$ : Hasemi et al Plume

M : MacCaffrey Plume

$\mathbf{Z}$ : Zukoski et al Plume

MZ : Modified Zukoski

Plume (in COMPBRN III)

Figure 2: Hot Gas Temperature for Experiment 1

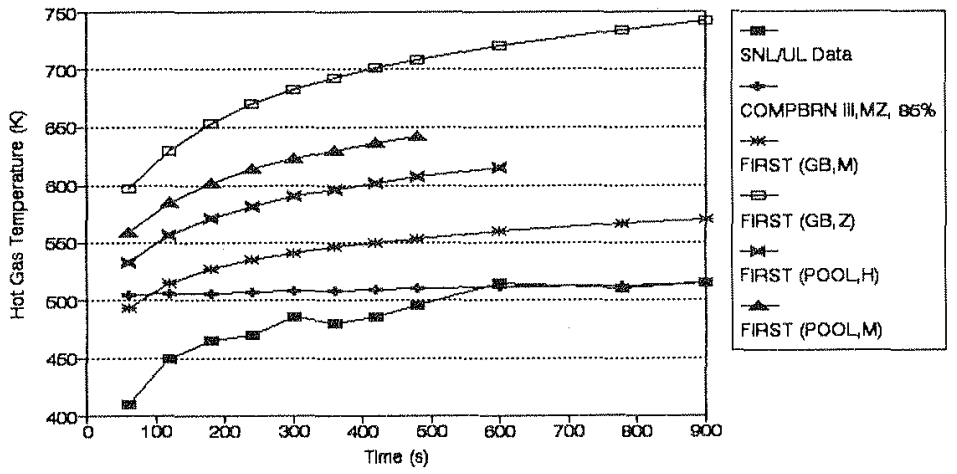

Figure 3: Hot Gas Temperature for Experiment 1

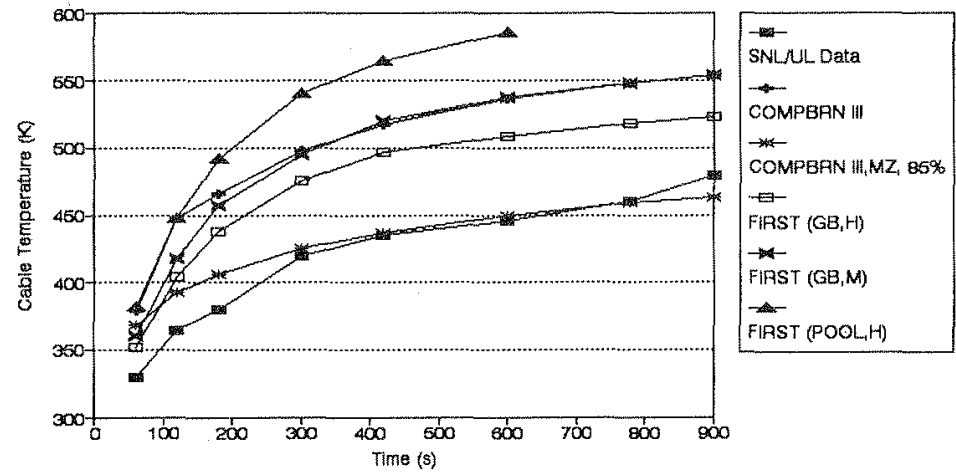

Figure 4: Cable Temperature for Experiment 1 


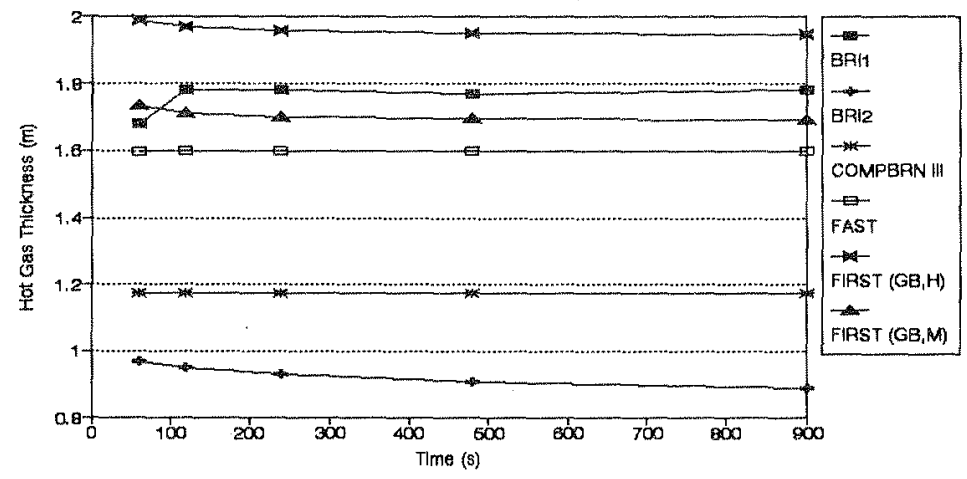

Figure 5: Hot Gas Thickness for Experiment 1

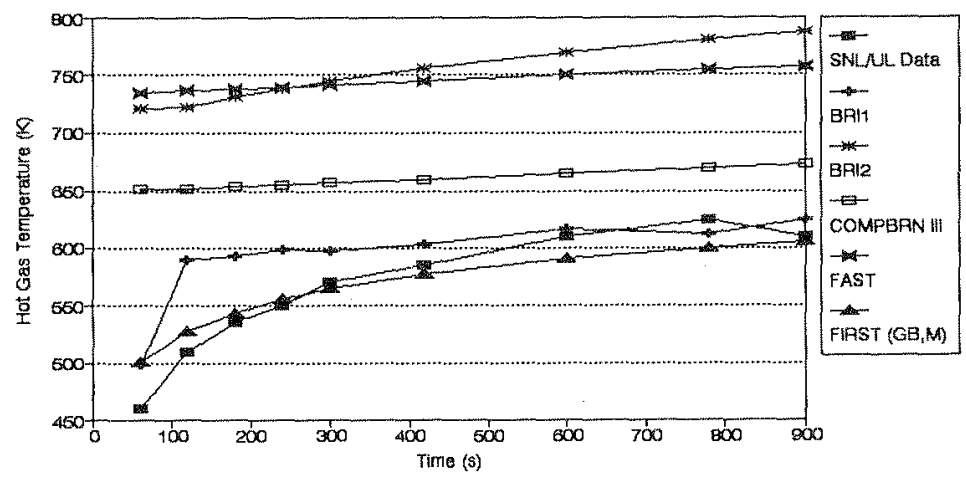

Figure 6: Hot Gas Temperature for Experiment 3

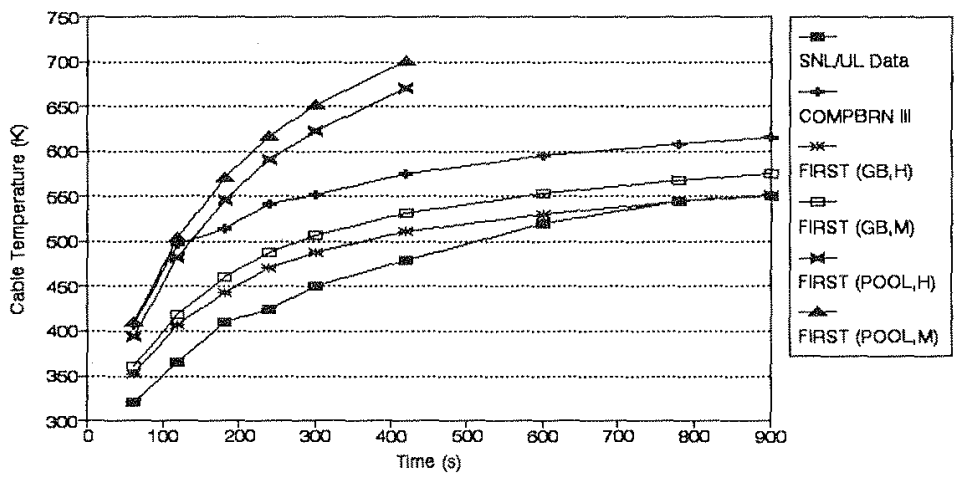

Figure 7: Cable Temperature for Experiment 3 


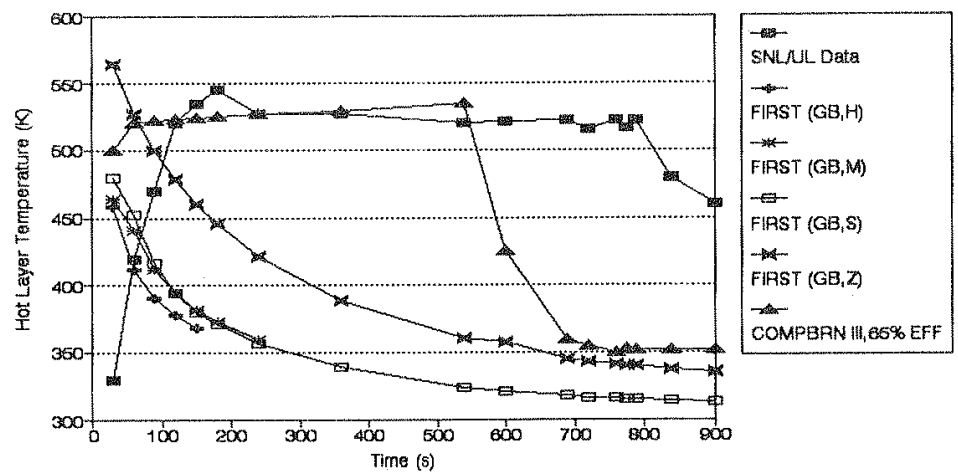

Figure 8: Hot Gas Temperature for Experiment 4

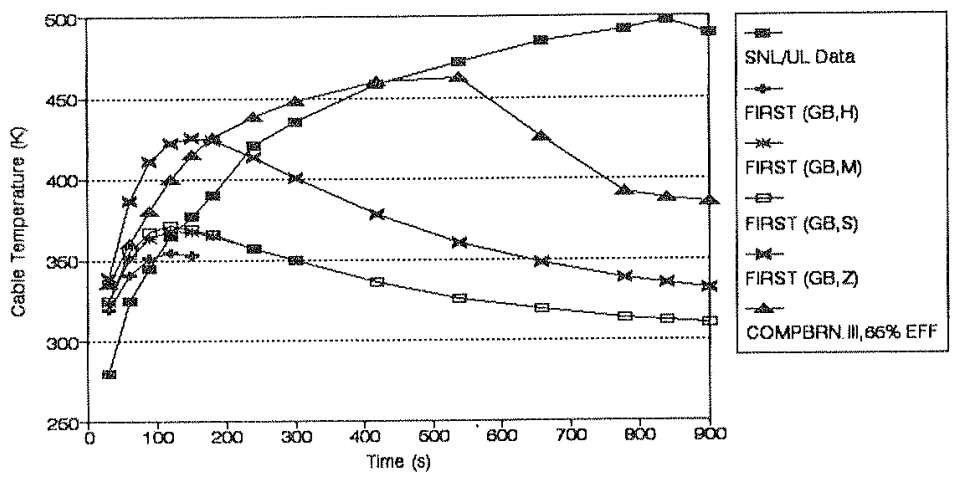

Figure 9: Cable Temperature for Experiment 4

\section{Model Accuracy}

As pointed out by Mitler [15], errors of about $20 \%$ are probably acceptable in view of the complex phenomena involved in fires. However, the errors in many cases (particularly FIRST's pool fires and BRI2) in this analysis are well outside that limit. The probable sources of error are likely to be the following:

Combustion Efficiency. Ho et al [4] carried out a number of simulations to determine combustion efficiency of heptane and they concluded that good agreement with experiments 1 and 3 was obtained with the value of 0.85 . In this study, all simulations used 0.97 [1], as 0.85 has been suggested as being applicable in under-ventilated situations [1]. Since FIRST, FAST, BRI2 and BRI1 all calculated the oxygen concentration at each time interval, their temperature curves would be more likely to represent the real behaviour of fires in a compartment (Figures 2 and 6). 
Rate of Heat Release. FIRST has been demonstrated [e.g 6,8] to be quite accurate for gas burner simulations but not for pool fires [8]. Therefore, COMPBRN III's

predictions of the heat release rate, which are consistent with [8], are used in this study .

Experimental errors. The measured values given by SNL/UL might contain some errors, possibly due to experimental technique and/or calibration.

Entrainment Model. The best entrainment model has not yet been established. As all models gave quite different results, further work is urgently required. In this study, Hasemi and McCaffrey plumes seem to give reasonable results, even though the Hasemi plume has been shown as perhaps not quite applicable for large fires [8].

Efrect of Fire Shape. The fires were burnt in a narrow, rectangular tray. Due to turbulence and eddy mixing at corners of the long tray, the fires would have entrained more air than necessary for full combustion, resulting in low hot gas temperatures in both experiments. In FIRST, all pool fires are assumed to be circular.

Pool Fire Model. The errors in FIRST's pool fire simulations are so high that the only explanation is that the treatment of relatively long and narrow fires as circular fires is not valid and the pool fire model in FIRST should probably be revised. Some results are shown in Figures 3, 4 and 7. Radiation feedback clearly plays a very important role. It increased the rate of heat release by $50 \%$ over and above that of the gas burner case. For heptane, Babrauskas [16] gives heat of vaporisation of $505.0 \mathrm{KJ} / \mathrm{kg}$, compared with Mitler and Rockett's $362.0 \mathrm{KJ} / \mathrm{kg}$ [1]. When Babrauskas' value is used, the results compare more favourably with the experimental data.

Data Adequacy. The purpose of the SNL/UL experiments was to examine hazardous situation associated with power plants. It might however, have been better if researchers had got involved from the early stage of experimental design and testing. This would have allowed maximum value to be obtained from the experiments, for both the original purpose as well as for modelling, at a minimum cost. For example, the mass loss rate of fuel and the thickness of hot layer were not recorded in these experiments. The data would have elucidated many points with regard to the validity of the experiments and computer simulations.

\section{CONCLUSIONS}

The comparison between the various models and the experimental results shows that:

- For the gas burner case, there is good agreement between the SNL/UL experiments and FIRST (Hasemi and McCaffrey plumes), BRI1 and COMPBRN III. Further study of fire plumes, effects of fire shape on entrainment rate and pool fire characteristics are needed for improvement of prediction. 
- There is clearly a need to have close cooperation between fire researchers and testing personnel to achieve better understanding of fires in buildings and to reduce hazards. It is hoped that this will be the case in future experiments.

\section{REFERENCES}

1) Mitler, H.E, and Rockett, J.A, "User's Guide to FIRST, a Comprehensive SingleRoom Fire Model", NBSIR 87-3595, National Bureau of Standards, U.S.A.

2) Cooper, L.Y, and Stroup, D.W, "Calculating Available Safe Egress Time (ASET)A Computer Program and User's Guide", NBSIR 82-2578, National Bureau of Standards, U.S.A.

3) Bukowski, R.W, Peacock, R.D, Jones, W.W, and Forney, C.L, "Hazard I, Fire Hazard Assessment Method", NIST Handbook 146, Vol 1, 1989, National Institute of Standards and Technology, U.S.A.

4) Ho, V, Siu, N, and Apostolakis, G, "COMPBRN III-A Fire Hazard Model for Risk Analysis", Fire Safety J, 13(1988)137.

5) Tanaka, T, "A Model of Multiroom Fire Spread", NBSIR 83-2718, National Bureau of Standards, U.S.A.

6) Rockett, J.A, "Park Service Room Fire Test Simulations using the Harvard Level 5.2 Computer Fire Model", NBSIR 83-2805, National Bureau of Standards, U.S.A.

7) Peacock, R.D, Davis, S, and Lee, B.T, "An Experimental Data Set for the Accuracy Assessment of Room Fire Models", NBSIR 88-3752, National Bureau of Standards, U.S.A.

8) Duong, D.Q, "The Accuracy of Computer Fire Models: Some Comparisons with Experimental Data from Australia", Fire Safety J, Vol 16, 1990, pp 415-431.

9) Zukoski, E.E, Kubota, T, and Cetegen, B, "Entrainment in Fire Plumes", Fire Safety J, 3(1980/1981)107.

10) McCaffrey, B.J, and Quintiere, J.G, "Buoyancy-driven Countercurrent Flows generated by a Fire Source", Heat Transfer and Turbulent Buoyant Convection, Vol 2, Hemisphere Publishing Co 1977,457.

11) McCaffrey, B.J, "Momentum Implications for Buoyant Diffusion Flames", Combustion and Flames, 52(1983)149.

12) Tokunaga, T, Sakai, T, Kawagoe, K, Tanaka, T, and Hasemi, Y, "Mass Flow Rate Formula for the Upward Current above Diffusion Flames", Fire Science and Technology, 2(1982)117.

13) Morton, B.R, Taylor, G, and Turner, J.S, "Turbulent Gravitational Convection from Maintained and Instantaneous Sources", Proceedings of the Royal Society (London), A234, pp 1-23, 1956.

14) Ho, V, Private Communication, Sept 1990.

15) Mitler, H.E, "Comparison of Several Compartment Fire Models: An Interim Report", NBSIR 85-3233, National Bureau of Standards,U.S.A.

16) Babrauskas, V, "Estimating Large Pool Fire Burning Rates", Fire Technology 19 (1983), pp 251-261. 